\title{
PLANTS
}

\section{A FLORISTIC INVENTORY OF THE MCLENNAN LAKE AREA, SASKATCHEWAN}

\section{DIANA BIZECKI ROBSON, 811 Avenue E North, Saskatoon, SK S7L 1S7}

In June of 19981 was able to explore the McLennan Lake area, north of La Ronge, Saskatchewan. This part of the province is in the Boreal Shield ecoregion. ${ }^{2}$ The Shield is much different in appearance than the Boreal Plain ecoregion to the south, which is covered by thick glacial till. The Boreal Shield has greater topographical changes, and exposed Precambrian rock outcrops and cliffs. The soils are thin relative to those in the Boreal Plain, especially near tops of hills. While exploring rock outcrops it often amazed me that there was such a diversity of flora growing in such little soil.

Black spruce (Picea glauca) and trembling aspen (Populus tremuloides) dominate the forests in the McLennan Lake area. Jack pine (Pinus banksiana) is the other most common tree -species. White spruce (Picea glauca) and tamarack (Larix laricina), while present, are relatively uncommon. An incredible diversity of understory vegetation is present including attractive mosses and lichens too numerous to count. Dry ground cranberry (Vaccinium vitis-idaea

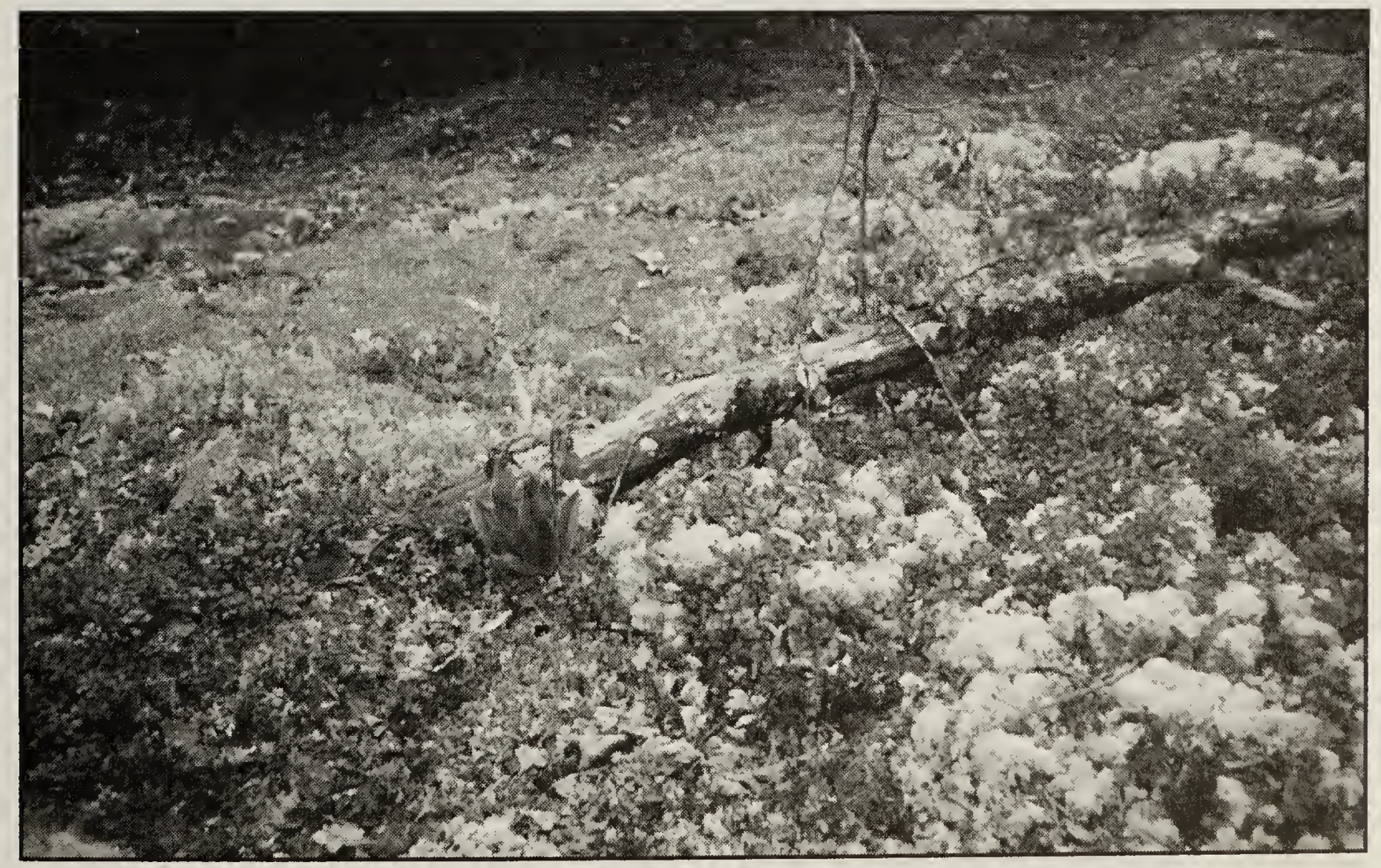

Figure 1 - Stemless lady's-slipper and associated habitat

Diana Bizecki Robson 


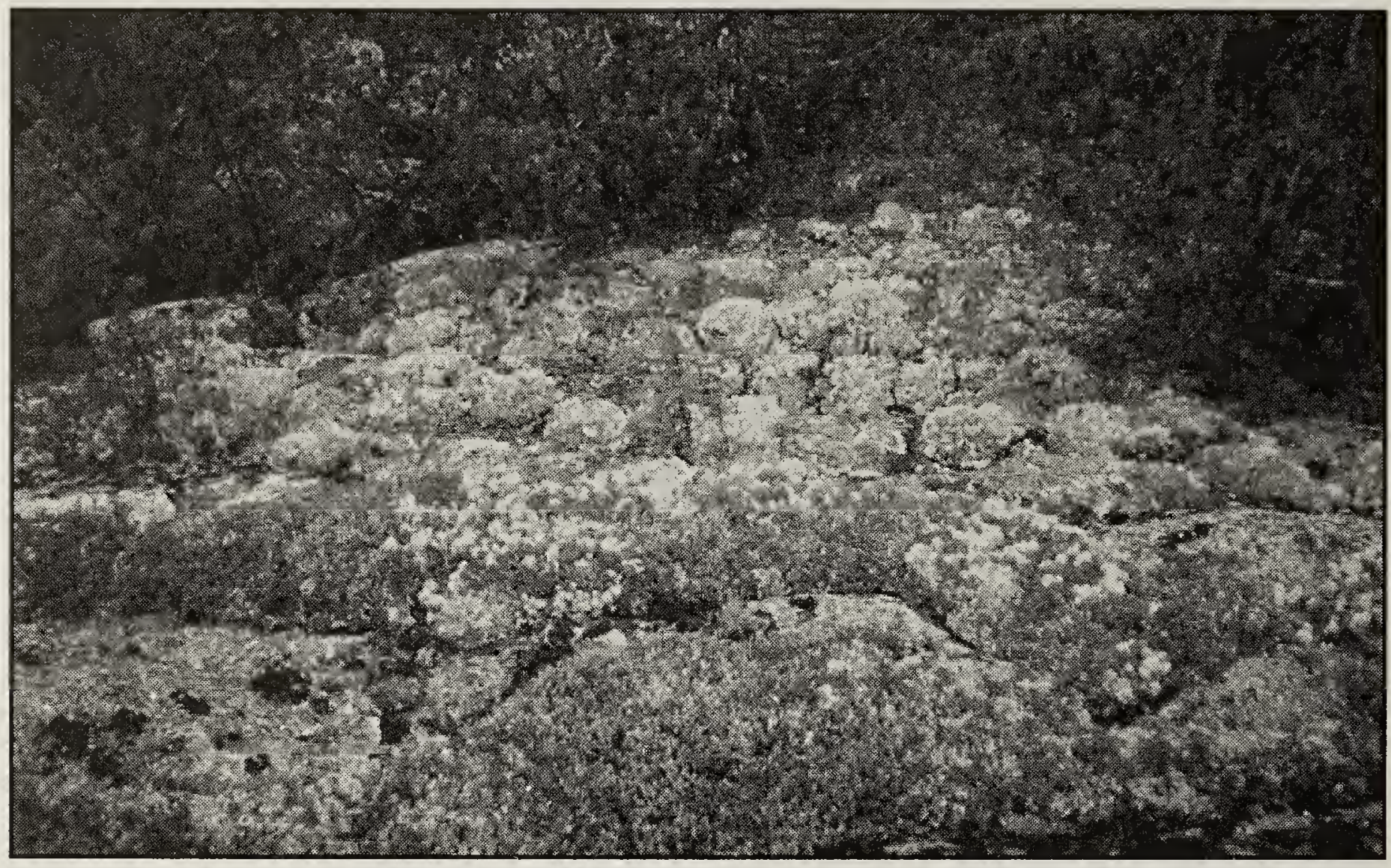

Figure 2 - Lichen-covered rock outcrop

Diana Bizecki Robson

var. minor) is the most common understory plant. This species produces large quantities of berries that are an important food source for mammals indigenous to the area like the Black Bear and Western Chipmunk. Other common understory vascular plants are club mosses (Lycopodium spp.), wintergreens (Pyrola spp.), bunchberry (Cornus canadensis), cloudberry (Rubus chamaemorus) and western Canada violet (Viola rugulosa). The beautiful stemless lady's-slipper (Cypripedium acaule) can sometimes be found in open jack pine stands among the reindeer moss (Cladina spp.) and dry ground cranberry (Figure 1). In ravines connecting lakes together, beautiful communities of ferns, (three of them considered rare in the province), berry-producing shrubs and sweet scented mints abound.

By far the most amazing places in the area are the granitic cliffs and hill topsthey are a lichen lover's paradise. Crustose (crust-forming), foliose (leafforming) and fruticose (branchlike) lichens of yellow, orange, brown, black and green cover nearly every square inch of rock (Figure 2). Hardy vascular plants like three-toothed saxifrage (Saxifraga tricuspidata), blueberry (Vaccinium angustifolium var. myrtilloides), bristly sarsaparilla (Aralia hispida), rusty woodsia (Woodsia ilvensis) and parsley fern (Cryptogramma acrostichoides) grow in tiny pockets of soil present in the rock cracks of cliffs (Figure 3 ).

My floristic survey revealed that there were 109 species of vascular plants present in the area. However, there are likely more species present since later blooming plants would not have been visible when I visited the area. Thus, this list should not be considered complete; merely a starting point for further study. Some of the moss and lichen genera and species discovered while exploring this area were also recorded. Since only a brief study of the bryophytes and lichens was conducted many more species are likely present.

All vascular plant species were collected, except those marked with an asterisk, and were donated to the W.P. 


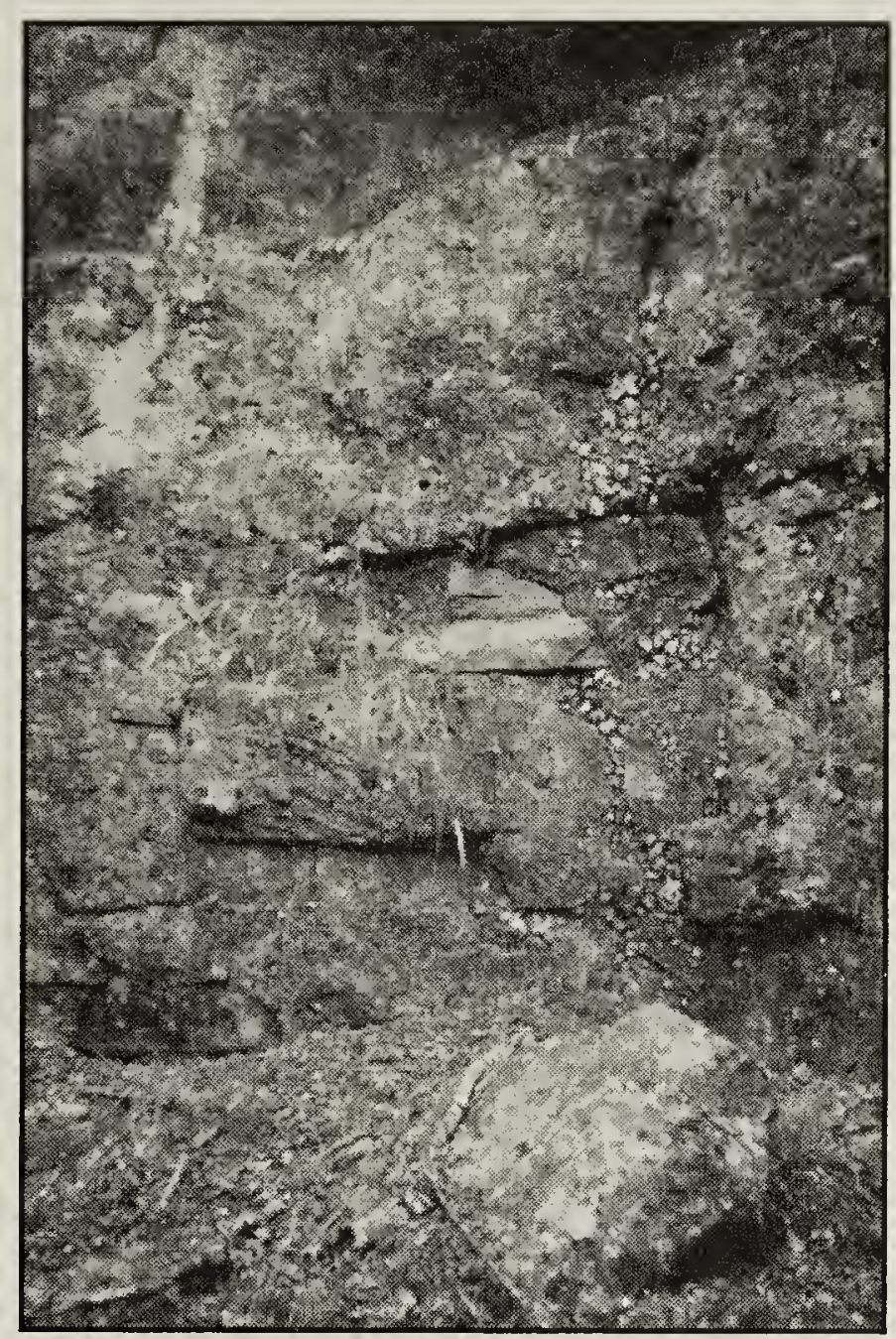

Figure 3 - A lichen and fern covered cliff

Diana Bizecki Robson

Fraser Herbarium at the University of Saskatchewan in Saskatoon. The lichen specimens were donated to Dr. John Sheard's lichen herbarium at the University of Saskatchewan. Nomenclature follows Kartesz and Kartesz. ${ }^{1}$ Plants considered rare by the Saskatchewan Conservation Data Centre are in bold-faced type.

\section{CHECKLIST OF THE FLORA OF THE MCLENNAN LAKE AREA}

\section{PLANTAE - PLANT KINGDOM} TRACHEOPHYTA - Vascular Plants

PTERIDOPHYTA - Ferns and Fern Allies

POLYPODIACEAE - Fern Family Athyrium filix-femina (L.) Roth - Lady fern

Cryptogramma acrostichoides R.Br. Parsley fern
Cystopteris fragilis (L.) Bernh. - Fragile fern

Dryopteris cristata (L.) Gray - Crested shield fern

Dryopteris phegopteris (L.) Schott Beech-fern

Gymnocarpium dryopteris (L.) Newm. Oak fern

Polypodium virginianum (L.) D.C. Eaton

- Common rock tripe

Woodsia ilvensis (L.) R.Br. - Rusty woodsia

EQUISETACEAE - Horsetail Family

Equisetum hyemale L. - Common scouring-rush

Equisetum sylvaticum L. - Woodland horsetail

LYCOPODIACEAE - Club-moss Family Lycopodium annotinum L. - Stiff clubmoss

Lycopodium clavatum L. - Running-pine Lycopodium complanatum L. - Trailing club-moss

Lycopodium obscurum L. - Ground-pine

SPERMATOPHYTA - Seed-bearing Plants

\section{TREES}

PINACEAE - Pine Family

Larix laricina (DuRoi) K. Koch Tamarack *

Picea glauca (Moench) Voss - White spruce *

Picea mariana (Mill.) BSP.- Black spruce Pinus banksiana Lamb. - Jack pine SALICACEAE - Willow Family Populus balsamifera L. - Balsam poplar Populus tremuloides Michx, Trembling aspen

BETULACEAE - Birch Family

Betula occidentalis Hook. - River birch Betula papyrifera Marsh. - White birch

SHRUBS

CUPRESSACEAE - Cypress Family Juniperus communis L. - Low juniper BETULACEAE - Birch Family 
Alnus viridis (Chaix) DC. - Green alder MYRICACEAE - Bayberry Family Myrica gale L. - Sweet gale

CORNACEAE - Dogwood Family

Cornus sericea L. - Red-osier dogwood ERICACEAE - Heath Family

Arctostaphylos uva-ursi (L.) Spreng. Bearberry

Chamaedaphne calyculata (L.) Moench. - Leatherleaf

Gaultheria hispidula (L.) Muhl. Creeping snowberry

Kalmia polifolia Wang. - Pale laurel

Ledum groenlandicum Oeder Labrador-tea

Oxycoccus microcarpus Turcz. - Small bog cranberry

Vaccinium vitis-idaea L. var. minus Lodd. - Dry ground cranberry

Vaccinium angustifolium Ait. var. myrtilloides (Michx.) House - Blueberry

CAPRIFOLIACEAE - Honeysuckle Family

Viburnum edule (Michx.) Raf - Low bush-cranberry

Viburnum opulus L. var. americanum (Mill.) Ait. - High bush-cranberry ROSACEAE - Rose Family

Prunus pensylvanica L. - Pin cherry

Rosa acicularis Lindl. - Prickly rose Rubus idaeus L. - Wild red raspberry SAXIFRAGACEAE - Saxifrage Family Ribes hudsonianum Richards. Northern black currant

Ribes oxyacanthoides L. - Northern gooseberry

SALICACEAE - Willow Family

Salix bebbiana Sarg. - Beaked willow Salix discolor Muhl. - Pussy willow Salix monticola Bebb. - Mountain willow Salix pedicellaris Pursh - Bog willow

\section{HERBS}

TYPHACEAE - Cattail Family Typha latifolia L. - Common cattail

ARACEAE - Arum. Family Calla palustris $\mathrm{L}$. - Water calla
ORCHIDACEAE - Orchid Family

Cypripedium acaule Ait. - Stemless lady's-slipper

Goodyera repens var. repens (L.) Br. Lesser rattlesnake-plantain

LILIACEAE - Lily Family

Maianthemum canadense Desf - Twoleaved Solomon's-seal

SANTALACEAE - Sandalwood Family Geocaulon lividum (Richards.) Fern Northern bastard toad-flax

POLYGONACEAE - Buckwheat Family Polygonum cilinode Michx. - Bindweed RANUNCULACEAE - Crowfoot Family Ranunculus reptans L. - Creeping buttercup

CORNACEAE - Dogwood Family

Cornus canadensis L. - Bunchberry

ONAGRACEAE - Evening-primrose Family

Epilobium angustifolium L. - Fireweed

FUMARIACEAE - Furnitory Family

Corydalis sempervirens (L.) Pers. - Pink corydalis

MENYANTHACEAE - Buck-bean Family Menyanthes trifoliata L. - Buck-bean ARALIACEAE - Ginseng Family

Aralia hispida Vent. - Bristly sarsaparilla Aralia nudicaulis L. - Wild sarsaparilla RUBIACEAE - Madder Family

Galium trifidum L. - Small bedstraw LAMIACEAE - Mint Family

Lycopus virginicus $L$. var. pauciflorus Benth - Northern water-horehound Mentha arvensis L. - Wild mint LORANTHACEAE - Mistletoe Family Arceuthobium Bieb. spp. * - Mistletoe CARYOPHYLLACEAE - Pink Family Stellaria calycantha (Ledeb.) Bong Northern stitchwort.

POTAMOGETONACEAE - Pondweed Family

Potamogeton praelongus Wulf - Whitestem pondweed

PRIMULACEAE - Primrose Family

Trientalis borealis Raf - Northern Starflower

ROSACEAE - Rose Family

Comarum palustre L. - Marsh cinquefoil Potentilla norvegica L. - Rough 
cinquefoil

Rubus chamaemorus L. - Cloudberry Rubus pubescens Raf - Dewberry Sibbaldiopsis tridentata (Aiton) Rydb. Three-toothed cinquefoil

SAXIFRAGACEAE - Saxifrage Family Heuchera richardsonii R.Br - Alumroot Saxifraga tricuspidata Rottb. - Threetoothed saxifrage

VIOLACEAE - Violet Family

Viola canadensis L. - Western Canada violet

HYDROPHYLLACEAE - Waterleaf Family

Phacelia_franklinii (R.Br.) Gray Franklin's scorpionweed

NYMPHAEACEAE - Water-lily Family Nuphar lutea (L.) Sm. - Yellow pond lily LENTIBULARIACEAE - Bladderwort Family

Utricularia vulgaris L. - Greater bladderwort

HALORAGACEAE - Water-milfoil Family

Myriophyllum spicatum L. - Spiked water-milfoil

PYROLACEAE - Wintergreen Family Moneses uniflora (L.) Gray - Oneflowered wintergreen

Orthilia secunda (L.) House - One-sided wintergreen

Pyrola asarifolia Michx. - Pink wintergreen

Pyrola chlorantha Sw. - Greenishflowered wintergreen

CAPRIFOLIACEAE - Honeysuckle Family

Linnaea borealis L. - Twinflower

ASTERACEAE - Aster Family

Achillea millefolium L. - Woolly yarrow Antennaria parvifolia Nutt. - Smallleaved pussytoes

Leucanthemum vulgare Lam. - Ox-eye daisy

Matricaria discoidea DC. Pineappleweed

Petasites frigidus (L.) Fr. - Palmateleaved colt's-foot

Senecio pauperculus Michx. - Balsam groundsel

Solidago spathulata DC. - Mountain goldenrod

GRAMINOIDS - Grasses and Grasslike Plants

POACEAE - Grass Family Alopecurus aequalis Sobol. - Shortawned foxtail

Calamagrostis canadensis (Michx.) Beauv. - Marsh reed grass

Oryzopsis pungens (Torr.) Hitchc. Northern rice grass

Poa glauca Vahl - Glaucous blue grass Poa nemoralis L, Wood blue grass CYPERACEAE - Sedge Family Carex aquatilis Wahl. - Water sedge Carex atherodes Spreng. - Awned sedge

Carex brevior (Dewey) Mack. - Sedge Carex buxbaumii Wahl. - Brown sedge Carex canescens L. - Short sedge Carex deflexa Hornem. - Bent sedge Carex disperma Dewey - Two-seeded sedge

Carex leptalea Wahl. - Bristle-stalked sedge

Eriophorum brachyantherum Trautv. Close-sheathed cotton-grass

JUNCACEAE - Rush Family Juncus balticus Willd. - Baltic rush Juncus longistylis Torn - Long-styled rush

BRYOPHYTA - Non-vascular Plants (Mosses \& Liverworts)

\section{MOSSES}

Ceratodon spp. *

Dicranum spp. *

Hylocomium spp.

Pleurozium schreberi

Polytrichum spp.

Ptilium spp. *

Pylaisiella spp.

Sphagnum spp. * 
LIVERWORTS

Ptilidium spp *

MYCETEAE - FUNGI KINGDOM

LICHENS

Actinogyra muhlenbergii

Bryoria spp.

Cetraria spp.

Cladina mites

Cladina stellaris

Cladonia amaurocrea

Cladonia gracilis

Cladonia coccifera

Flavocetraria spp.

Hypnogymnia spp.

Nephroma bellum

Parmelia spp. *

Peltigera canina

Stereocaulon tomentosum

Umbilicaria deusta

Usnea spp.

Xanthoparmelia taractica

* = No voucher specimen collected Bold $=$ plants are rare according to the Saskatchewan Conservation Data Center.

\section{Acknowledgements}

Thanks are extended to Dr. Vernon Harms for assisting with verification of the vascular plant specimens. Dr. John Sheard graciously agreed to identify the lichen species collected. Rick Kolstad and Debbie Peters of Forest House Lodge acted as tour guides and hosts during my visit.

\section{Literature Cited}

1. KARTESZ, J.T., AND R. KARTESZ. 1980. A synonymized checklist of the vascular flora of the United States, Canada and Greenland. University of North Carolina Press, Chapel Hill.

\section{ACTON, D.F., G.A. PADBURY, AND} C.T. STUSHNOFF. 1998. The ecoregions of Saskatchewan. Canadian Plains Research Center, University of Regina, Regina.

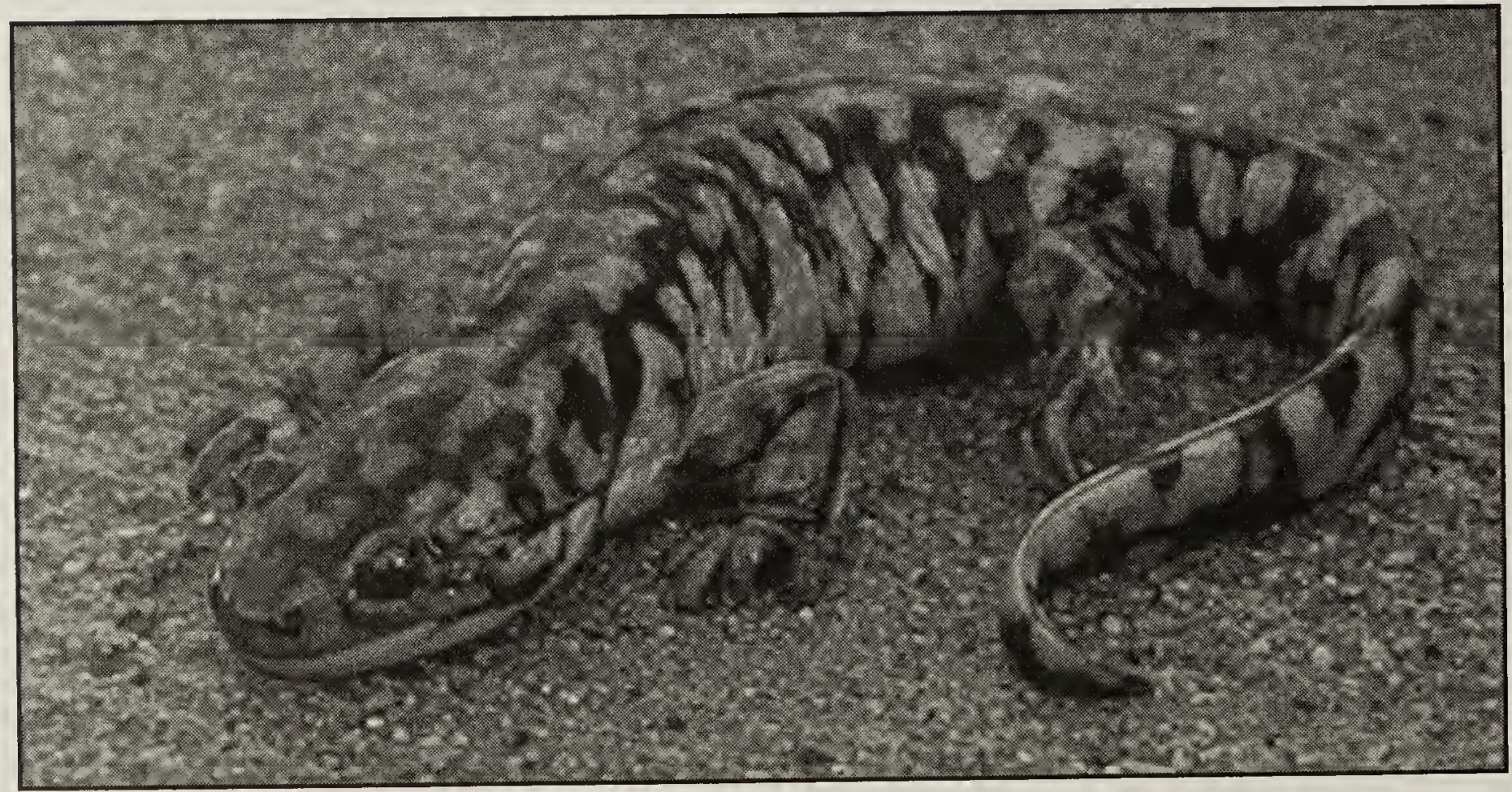

